\title{
CDS - Interfaces for published and reference data
}

\author{
Mark Allen ${ }^{1, \star}$ and the CDS Team \\ ${ }^{1}$ Université de Strasbourg, CNRS, Observatoire astronomique de Strasbourg, UMR 7550, F-67000 Stras- \\ bourg, France
}

\begin{abstract}
The Centre de Données astronomiques de Strasbourg (CDS) provides added value to published and reference data, enabling scientific research with the data through innovative services and tools. As a long standing data centre it is important to maintain and update the processes that have been developed over many years, in particular to manage the increasing volume of publications and data. CDS presentations at the LISA conference have highlighted these developments, and a poster supporting these presentations showed the current CDS user interfaces. Here we highlight how developments in the processes and underlying databases result in new features in the CDS user interfaces.
\end{abstract}

\section{Introduction}

The Centre de Données astronomiques de Strasbourg (CDS) is an astronomy reference data centre with the core role of providing added value to published and reference data, and enabling scientific research with the data through innovative services and tools. The processes for the ingestion of data into the CDS databases are constantly revised and adapted to new requirements and new technologies [1]. At this LISA meeting we have reported on evolutions, improvements and updates in the areas of:

- Cross-identification of astronomical objects for the SIMBAD database (Brunet)

- The new CDS documentalist task of curating pixel data in HiPS format (Buga)

- The VizieR associated data service for data (spectra, images etc.) connected to a journal article (Ocvirk)

- The certification of CDS services with the Data Seal of Approval (Landais)

- Shared nomenclature and identifiers for telescopes and instruments (Perret)

- The rapid development and future visions of technologies that facilitate the documentalits' work (Schaaff)

\section{Organisation of CDS work}

The CDS work is carried out by an integrated team of scientists, software engineers and documentalists with a deep level of interaction and coordination between the different types of activities. Figure 1 provides an overview of all of the activities of the CDS. The reference services are: SIMBAD, the

^e-mail: mark.allen@astro.unistra.fr ORCID: 0000-0003-2168-0087 
reference database for the nomenclature and bibliography of astronomical objects (outside the solar system) [2]. VizieR, the reference service for astronomical catalogues including tables and data published in academic journals [3], the Aladin interactive sky atlas, the CDS X-Match large astronomical catalogue cross-correlation service [4], and a new integrated CDS Portal ${ }^{1}$.

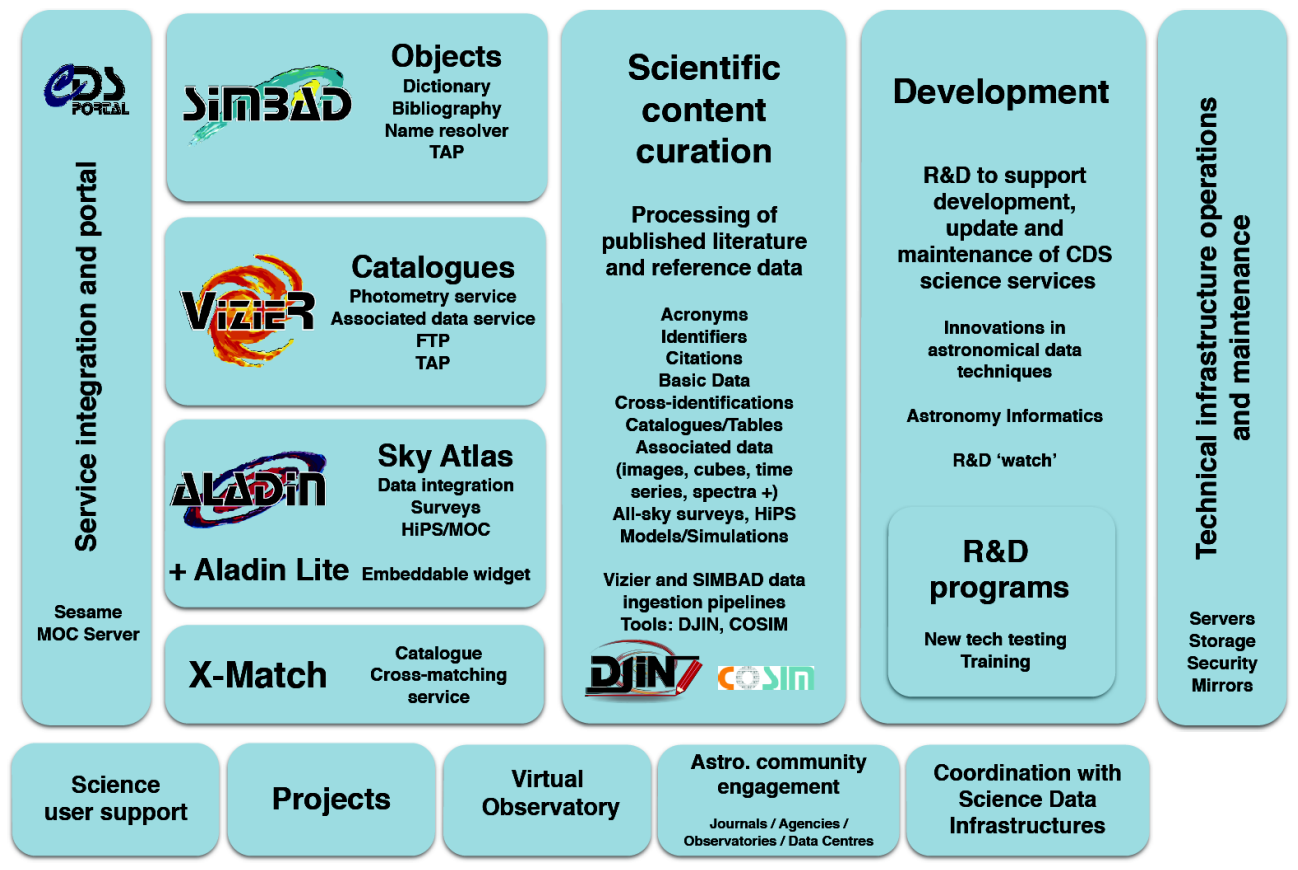

Figure 1. An overview of CDS activities

\section{CDS Service Interfaces}

Astronomers use the CDS services via the various interfaces. The CDS web pages ${ }^{2}$ provides the starting point for access to all of these interfaces, in particular the individual web interfaces of the different services. In addition to these web interfaces there are also many programmatic interfaces to CDS services that allow calls to these services by computer programs or scripts, which are often used by external services. For example, calls to the SIMBAD name resolver which is implemented in the search field of many observatory/mission archive services. Other examples include the access to CDS services via standard IVOA ${ }^{3}$ protocols such as the TAP (Table Access Protocol) interfaces to SIMBAD and VizieR that enables complex queries of these databases using the ADQL language, and also the SSA (Simple Spectral Access) and SIA (Simple Image Access) interfaces to the VizierR associated data service. The Aladin sky atlas has two main interfaces, one a desktop application, and the other called Aladin Lite [5] which is a javascript widget that can be embedded in web pages.

Recently CDS has released a new CDS Portal, which is an integrated interface to CDS databases, allowing uses to search across multiples services from a single query box. This has allowed us to

\footnotetext{
${ }^{1}$ http://cdsportal.u-strasbg.fr

${ }^{2}$ http://cds.unistra.fr

${ }^{3}$ International Virtual Observatory Alliance, http://ivoa.net
} 
explore and test new ideas for the interfaces, and to benefit from many improvements in the underlying database infrastructure and metadata.

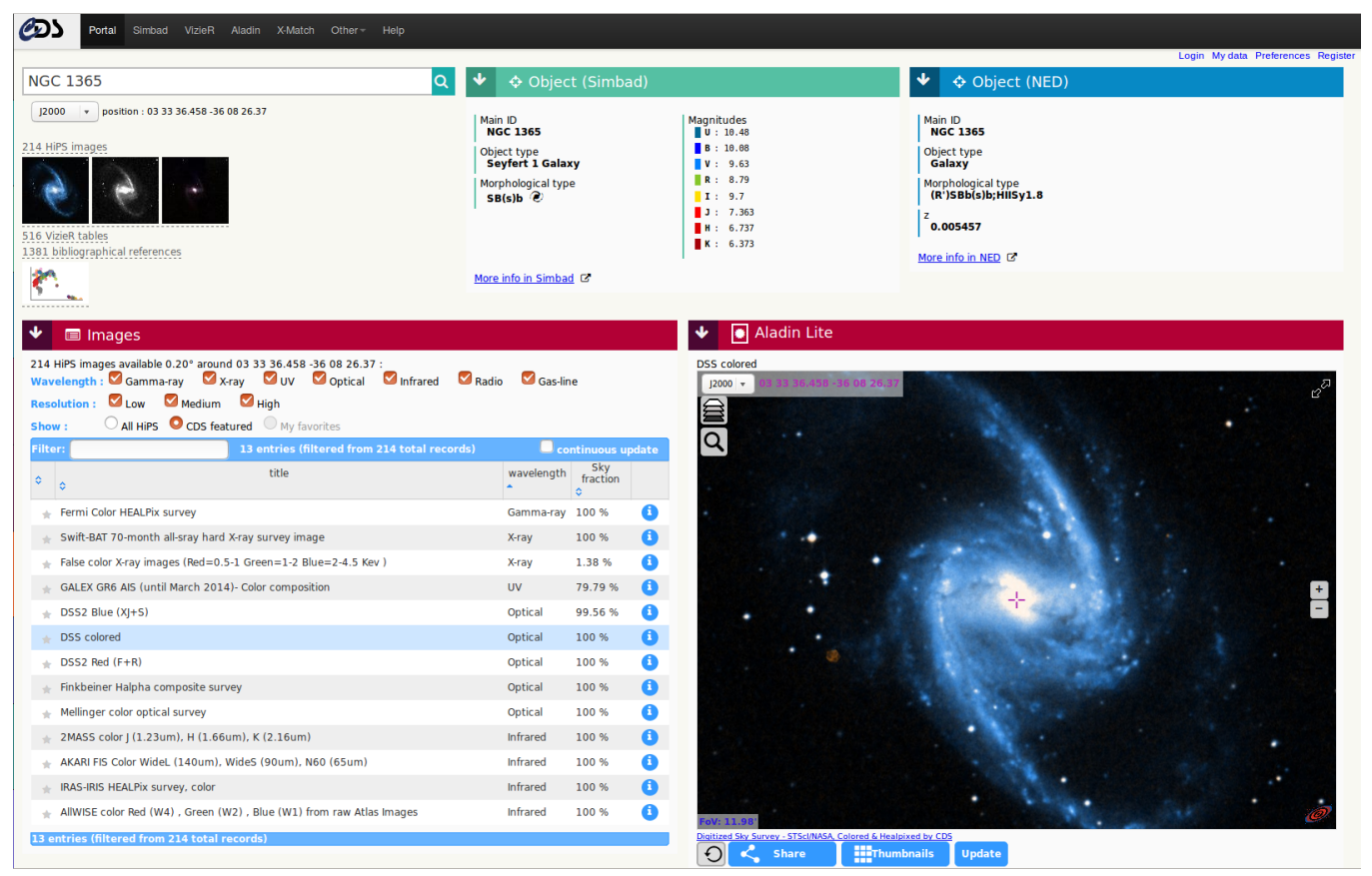

Figure 2. CDS Portal query results for Objects and Images

Figure 2 shows the result of query for information on the galaxy NGC 1365. Reference data from SIMBAD (and also NED) are provided with links to more details. The image section makes use of the new HiPS network of all-sky images [6], and its implementation in Aladin Lite. The metadata about the surveys allows for filtering of the hundreds of such surveys.

Figure 3 shows another part of the new CDS Portal for catalogues, with the result of the coordinate based query. The detailed metadata that is curated during the ingestion of catalogues into VizieR allows complex searching capabilities, with the new CDS Portal enabling interactive filtering via the use of facets. Searches can be constrained by wavelength, astronomy keywords, sky fraction and the presence of associated data files. Data may be selected and used for quick-look analysis in the portal including overlay on the Aladin Lite panel.

\section{Summary}

The reference data that is carefully ingested into the CDS services by the team of documentalists, engineers and astronomers, is made searchable and usable via the various interactive and programmatic interfaces. Continual adaptation and improvement in the ingestion processes is necessary for managing the increasing volume of data associated with journal publications, and to respond to the scientific needs of the astronomical community. Recent advances in object cross-identification, handling of very large pixel data sets and associated data, all contribute to the quality and interoperability of the reference data, allowing it to be used in innovative new ways. 


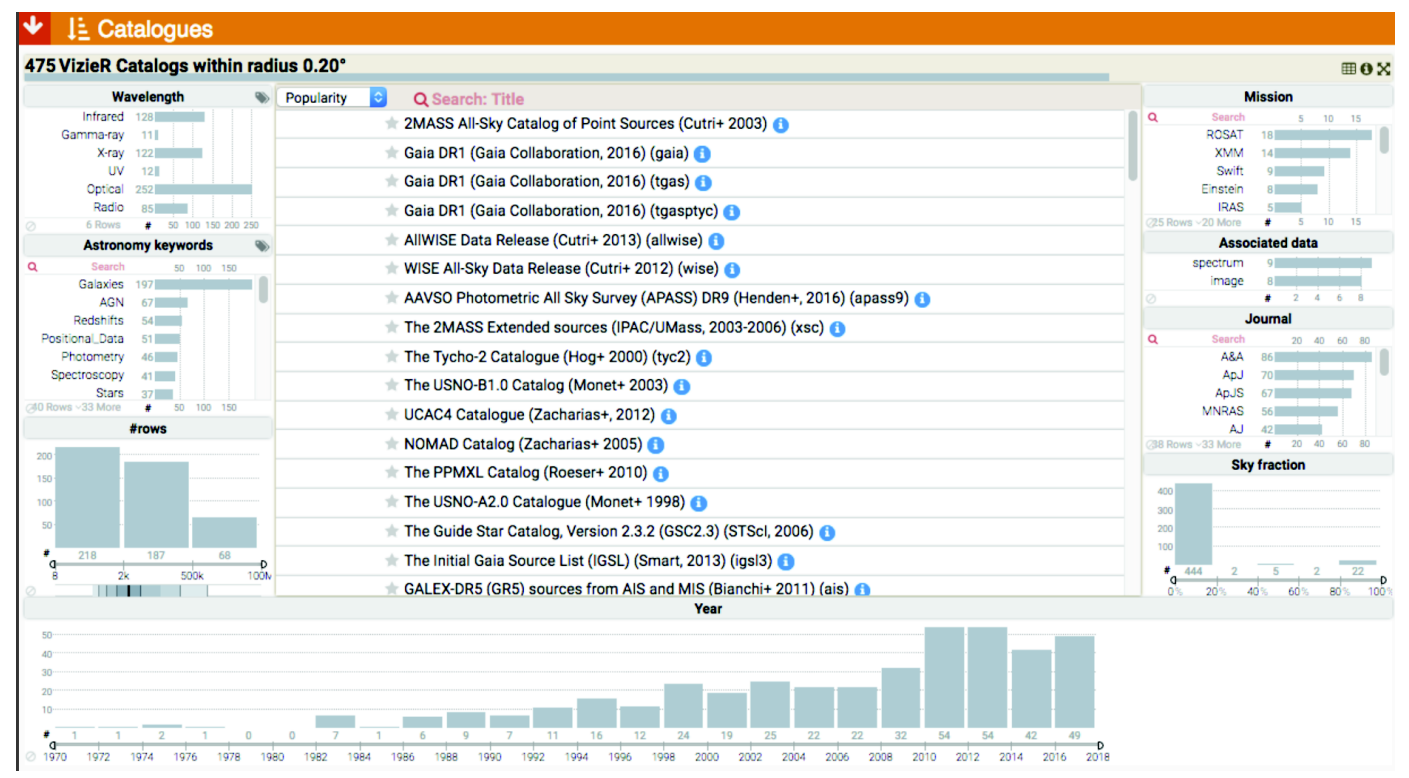

Figure 3. CDS Portal query results for Catalogues

\section{References}

[1] E. Perret, T. Boch, F. Bonnarel et al., Working Together at CDS: The Symbiosis Between Astronomers, Documentalists, and IT Specialists in Open Science at the Frontiers of Librarianship, edited by András Holl, Soizick Lesteven, Dianne Dietrich, and Antonella Gasperini (2015), Vol. 492 of Astronomical Society of the Pacific Conference Series, p. 13-21

[2] M. Wenger, F. Ochsenbein, D. Egret et al., A\&AS 143, 9 (2000)

[3] G. Landais, T. Boch, M. Brouty, et al., Management of Catalogs at CDS in Open Science at the Frontiers of Librarianship, edited by András Holl, Soizick Lesteven, Dianne Dietrich, and Antonella Gasperini (2015), Vol. 492 of Astronomical Society of the Pacific Conference Series, p. $57-60$

[4] F. Pineau, T. Boch, S. Derriere, \& Arches Consortium, Towards a Next-Generation Catalogue Cross-Match Service in Astronomical Data Analysis Software an Systems XXIV (ADASS XXIV), edited by A.R. Taylor and E. Rosolowsky (2015), Vol. 495 of Astronomical Society of the Pacific Conference Series, p. 61-64

[5] T. Boch, P. Fernique, Aladin Lite: Embed your Sky in the Browser in Astronomical Data Analysis Software and Systems XXIII (ADASS XXIII), edited by N. Manset and P. Forshay (2014), Vol. 485 of Astronomical Society of the Pacific Conference Series, p. 277-280

[6] P. Fernique, M. G. Allen, T. Boch, et al., A\&A 578, A114 (2015) 